\title{
ReaR
}

\section{Actualización de Analgesia epidural para el trabajo de parto.}

Artículo original: Onuoha OC. Epidural Analgesia for Labor: Continuous Infusion Versus Programmed Intermittent Bolus. Anesthesiol Clin. 2017;35(1):1-14. doi:10.1016/j.anclin.2016.09.003 (PubMed) Sánchez Quirós B, López Herrero R, Lomo Montero FJ.

\section{Hospital Clínico Universitario de Valladolid}

\section{Resumen}

El dolor del trabajo de parto produce no sólo sufrimiento emocional sino alteraciones fisiológicas que afectan a la madre y al feto tales como: hiperventilación, alcalosis respiratoria severa aumento de catecolaminas y hormonas de estrés, hipoxia feto-placentaria y acidosis fetal y alteraciones psicológicas y estrés postraumático.

Actualmente, la anestesia neuroaxial y más concretamente la analgesia epidural es la principal recomendación como método farmacológico de analgesia en el trabajo de parto. Nos hemos centrado principalmente en dos métodos de infusión. Por un lado, el tradicional, de infusión continua de anestésico local por el catéter epidural (CEI) más bolos de analgesia epidural controlada por el paciente (PCEA) y por otro; epidural con bolos intermitentes programados: (PIEB) que es un método de infusión de fármacos en el espacio epidural que consiste en la administración de bolos fijos en intervalos determinados.

\section{Introducción}

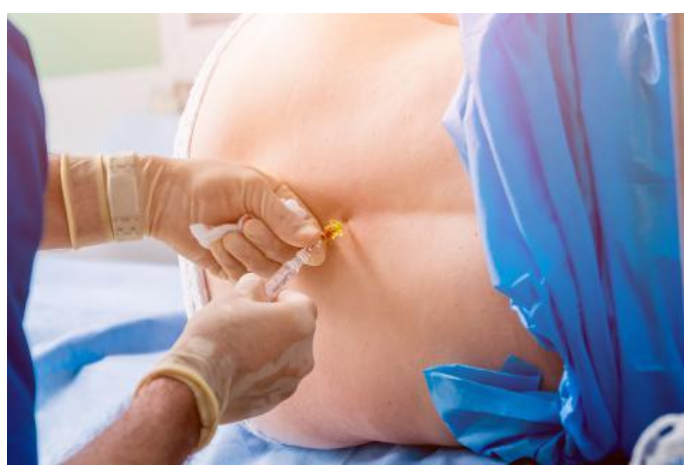

El dolor del trabajo de parto produce no sólo sufrimiento emocional sino alteraciones fisiológicas que afectan a la madre y al feto tales como: hiperventilación, alcalosis respiratoria severa aumento de catecolaminas y hormonas de estrés, hipoxia fetoplacentaria y acidosis fetal y alteraciones psicológicas y estrés postraumático.
Actualmente, la anestesia neuroaxial y más concretamente la analgesia epidural es la principal recomendación como método farmacológico de analgesia en el trabajo de parto. Nos hemos centrado principalmente en dos métodos de infusión.

Por un lado, el tradicional, de infusión continua de anestésico local por el catéter epidural (CEI) más bolos de analgesia epidural controlada por el paciente (PCEA) y por otro; epidural con bolos intermitentes programados: (PIEB) que es un método de infusión de fármacos en el espacio epidural que consiste en la administración de bolos fijos en intervalos determinados.

El dolor del trabajo de parto produce no sólo sufrimiento emocional sino alteraciones fisiológicas que afectan a la madre $y$ al feto tales como: hiperventilación, alcalosis respiratoria severa aumento de catecolaminas $\mathrm{y}$ 
hormonas de estrés, hipoxia fetoplacentaria, acidosis fetal, alteraciones psicológicas y estrés postraumático.

Actualmente, la anestesia neuroaxial y más concretamente la analgesia epidural es la principal recomendación como método farmacológico de analgesia en el trabajo de parto. Nos hemos centrado principalmente en dos métodos de infusión. Por un lado, el tradicional, de infusión continua de anestésico local por el catéter epidural (CEI) más bolos de analgesia epidural controlada por el paciente (PCEA) y por otro; epidural con bolos intermitentes programados: (PIEB) que es un método de infusión de fármacos en el espacio epidural que consiste en la administración de bolos fijos en intervalos determinados.

Se analizaron cuatro ensayos clínicos aleatorizados que comparaban ambas técnicas con distintos tipos, volúmenes y concentraciones de anestésico local. Se determinó que la técnica de bolos automáticos es superior a la técnica de infusión continua, proveyendo igual o mejor analgesia con menor dosis de fármaco. Disminuye el bloqueo motor y consecuentemente, disminuye la incidencia de parto instrumentado y las complicaciones asociadas.

El mecanismo responsable de esta diferencia no es bien conocido. Las disecciones en cadáveres han mostrado una mayor extensión del líquido en el espacio epidural, a través de los agujeros intervertebrales cuando usamos grandes volúmenes inyectados a alta presión. Esto sugiere que las soluciones epidurales se distribuyen mejor cuando son inyectadas en bolo que en perfusión continua.

\section{Introducción}

La SEGO (Sociedad Española de Ginecología y Obstetricia) considera que la atención al parto debe basarse en los principios de humanización, control fetal y alivio del dolor. (1).

El requerimiento materno es una indicación suficiente para la administración de analgesia epidural. El inicio precoz no aumenta el riesgo de cesárea ni ocasiona riesgos adicionales. Existen diversas condiciones, en las que el beneficio de la epidural sobre otras técnicas especialmente alto y por lo tanto su utilización fuertemente recomendada. En casos de pacientes de riesgo alto para la anestesia general (vía aérea difícil, obesidad mórbida, etc.) es recomendable la inserción precoz $\mathrm{y}$ efectiva de un catéter epidural. A continuación vamos a analizar las mejores estrategias de analgesia epidural (2)

\section{Material y Métodos}

Se analizaron cuatro ensayos clínicos, aleatorizados a doble ciego:

\section{ESTUDIOS}

\section{Wong and colleagues (3)}

Anestesia neuroaxial combinada con bolo inicial de bupivacaína $1.25 \mathrm{mg}+15$ microgramos de fentanilo. Dos grupos:

$$
\begin{aligned}
& \text { 1. + PIEB (Bolos 6ml cada 30min) } \\
& \text { +PCEA } \\
& \text { 2. +CEI }(12 \mathrm{ml} / \mathrm{h})+\text { PCEA }
\end{aligned}
$$

\section{Fettes and colleagues (4)}

Bolo epidural inicial de Ropivacaína $0.2 \%$ de entre 15 y $20 \mathrm{ml}$ hasta conseguir analgesia y bloqueo sensorial hasta T10, después eran aleatoriamente asignados a dos grupos:

1. Infusión continua de
Ropivacaína $0.2 \%$ a $10 \mathrm{ml} / \mathrm{h}$

2. Bolo intermitente (Bolo de 10 ml cada hora) 


\section{Capogna and colleagues (5)}

Bolo epidural inicial de Levobupivacaína $\quad 0.0625 \% \quad 20 \mathrm{~mL}+$ 10microgramos de sufentanilo:

1. PIEB $(10 \mathrm{~mL}$ de levobupivacaína $0.0625 \%$ cada hora) + PCEA

2. CEI $10 \mathrm{~mL} / \mathrm{h}+$ PCEA

\section{Wong and colleagues (2011) (6)}

Extendieron la PIEB tanto el volumen como el intervalo, de $2.5 \mathrm{ml}$ cada 15 minutos a $10 \mathrm{ml}$ cada hora.

\section{Resultados}

Un resumen de los principales resultados obtenidos de estos estudios se muestra en las tablas 1 y 2 .

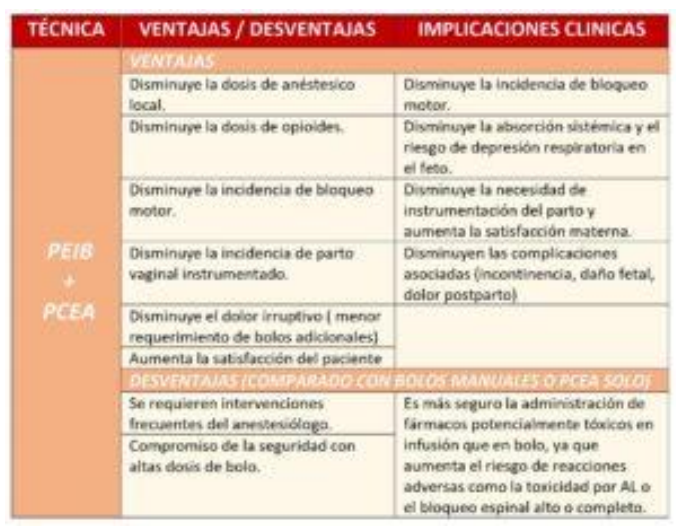

Tabla 1. Ventajas y desventajas de la administración de bolo epidural intermitente (PEIB). Modificado de Onuoha OC. Epidural Analgesia for Labor: Continuous Infusion Versus Programmed Intermittent Bolus. Anesthesiol Clin. 2017;35(1):1-14

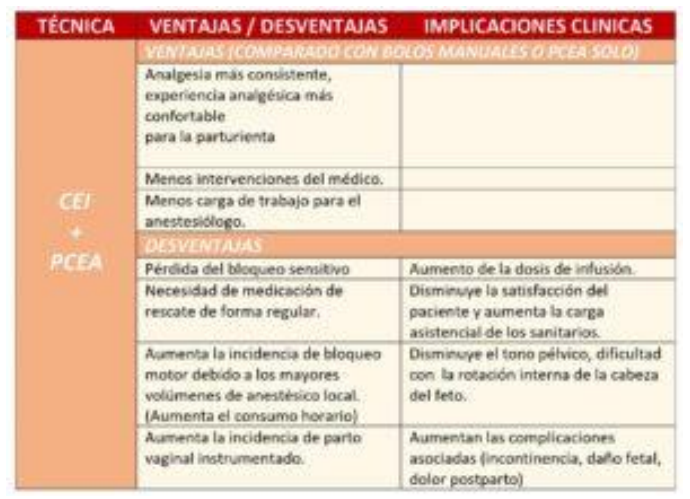

Tabla 2. Ventajas y desventajas de la administración de infusión continua (CEI).
Modificado de Onuoha OC. Epidural Analgesia for Labor: Continuous Infusion Versus Programmed Intermittent Bolus. Anesthesiol Clin. 2017;35(1):1-14

\section{Comentario}

Los estudios revelaron que la dosis de fentanilo fue menor también en el grupo de PIEB y que a satisfacción subjetiva con el manejo del dolor fue mayor en el grupo de bolo intermitente (3). También se observó que el tiempo hasta el primer rescate fue mayor en el PEIB (4), así como que el mantenimiento de la analgesia con PIEB, tuvo menos incidencia de bloqueo motor e instrumentación del parto comparado con CEI (5).

Este último punto es especialmente importante para el anestesiólogo, ya que al disminuir el número de cesáreas $\mathrm{y}$ partos instrumentados se evitan también las complicaciones derivadas de estas técnicas. Se concluyó también que extendiendo la PIEB, disminuía el consumo de bupivacaina sin disminuir la satisfacción subjetiva.

La diferencia no fue clínicamente significativa en bloqueo motor, pese a que los bolos eran de mayor volumen y en intervalos más cortos debido a que la concentración del bolo era menor, soluciones tan diluidas producían mínimamente o nulo bloqueo motor (6).

En base a estos resultados, se puede establecer un protocolo de administración de analgesia epidural en función de la opción analgésica que elijamos. El objetivo es conseguir la mayor potencia analgésica con el menor bloqueo motor.

En la Figura 1 se muestra el protocolo utilizando dos concentraciones de bupivacaina. El protocolo de bupivacaína a $0.125 \%$ podría sustituirse 
por ropivacaina $0.2 \%$ con similares efectos.

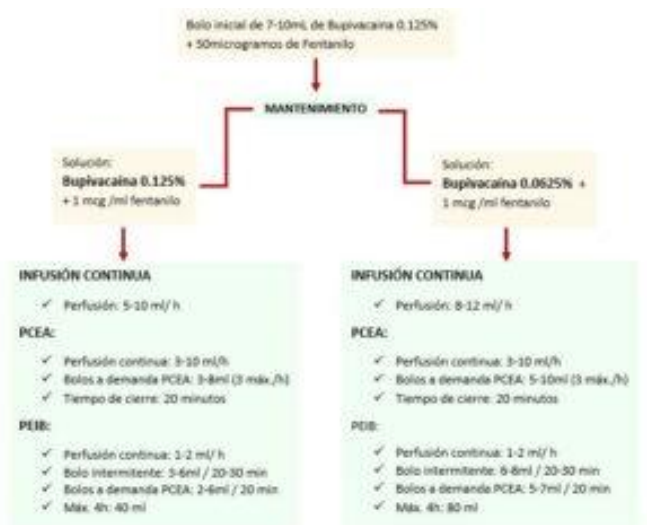

Figura 1. Protocolo de administración de analgesia epidural utilizando bupivacaina en dos concentraciones diferentes y en ambas modalidades.

\section{Bibliografía}

(1) Actualización de los protocolos asistenciales de la Sección de Anestesia Obstétrica de la SEDAR. 2 ${ }^{\mathrm{a}}$ Edición. 2016 (PDF)

(2) Arendt, Katherine W. MD The 2015 Gerard W. Ostheimer Lecture: What's New in Labor Analgesia and Cesarean Delivery, Anesthesia \& Analgesia: May 2016 - Volume 122 - Issue 5 p 1524-1531 doi: 10.1213/ANE.0000000000001265 (ㄴML)

(3) Wong CA, Ratliff JT, Sullivan JT, Scavone $\mathrm{BM}$, Toledo P, McCarthy RJ. A randomized comparison of programmed intermittent epidural bolus with continuous epidural infusion for labor analgesia. Anesth Analg.
2006;102(3):904-909.

doi:10.1213/01.ane.0000197778.57615.1a (

(4) Fettes PD, Moore CS, Whiteside JB, McLeod GA, Wildsmith JA. Intermittent vs continuous administration of epidural ropivacaine with fentanyl for analgesia during labour. Br J Anaesth. 2006;97(3):359-364. doi:10.1093/bja/ael157 (PDF)

(5) Capogna G, Stirparo S. Techniques for the maintenance of epidural labor analgesia. Curr Opin Anaesthesiol. 2013;26(3):261-267. doi:10.1097/ACO.0b013e328360b069 (PubMed)

(6) Wong CA, McCarthy RJ, Hewlett B. The effect of manipulation of the programmed intermittent bolus time interval and injection volume on total drug use for labor epidural analgesia: a randomized controlled trial. Anesth Analg. $\quad 2011 ; 112 \quad$ (4):904-911. doi:10.1213/ANE.0b013e31820e7c2f (HTML)

Correspondencia al autor

Belén Sánchez Quirós

belensq93@gmail.com

MIR Anestesiología y Reanimación.

Hospital Clínico Universitario de Valladolid.

Francisco Javier Lomo Montero

flomo@saludcastillayleon.es

FEA Anestesiología y Reanimación. Hospital Clínico Universitario de Valladolid

Aceptado para el blog en febrero de 2021 